\title{
Research Study on Target Treatment of Ultrasound Microbubbles with Docetaxel on Osteosarcoma
}

\author{
Dan Fei, Yang Lu, Guoqing Sui a, ${ }^{*}$ \\ Department of Ultrasound, China-Japan Union Hospital, of Jilin University, China. \\ *, a suigq15@mails.jlu.edu.cn
}

Keywords: Ultrasound; Micro bubble; Docetaxel; Osteosarcoma.

\begin{abstract}
Objective To investigate the therapeutic effect of target treatment on osteosarcoma cells through ultrasound micro bubble imaging with docetaxel. Methods The osteosarcoma model of Sprague-Dawley rats was established by implantation of tissue mass of UMR-106 cell line on the back. The model rats were randomly divided into 3 groups after modeling: the group of ultrasound micro bubbles with docetaxel, the group of docetaxel, and the control group. Three groups were given drugs once a week and stopped after 5 weeks of administration. The tumor mass weight was taken out to calculate the tumor inhibition rate within 24 hours after drug withdrawal. The difference of the parameters among the groups was analyzed by SPSS17.0 software. Results Targeted treatment of ultrasound micro bubble imaging with docetaxel could significantly inhibit the growth of osteosarcoma, and the inhibition rates of each experimental group were $24.2 \%$ and $12.1 \%$, respectively, indicating its significant function on inhibiting the growth of osteosarcoma cells. Compared with the experimental groups, the tumor inhibition rates among the experimental groups were statistically significant $(\mathrm{P}<0.05)$. Conclusion Docetaxel has a significant function on inhibiting the growth of osteosarcoma. Target treatment of ultrasound micro bubbles with docetaxel is conducive to improve the therapeutic effect and further achieve the purpose of targeted therapy.
\end{abstract}

\section{Introduction}

Osteosarcoma is a primary malignant tumor originating from mesenchymal tissue, accounting for $20 \%$ of the primary malignant tumors. It is prevalent in adolescents and can produce spindle like stromal cells of osteoid tissue, posing a great threat to the life and quality of patients. [1.2] With the development of chemotherapeutic drugs in the treatment of osteosarcoma, the emphasis of experimental research is what kind of chemotherapeutic drugs should be used and how to achieve the best therapeutic effect. This study investigates the efficacy of the new chemotherapeutic drug, docetaxel, in the treatment of osteosarcoma in recent years.

\section{Data and Methods}

Animal models Using Sprague-Dawley rats as animal model, healthy rats with the average age of 5-6 weeks and the body weight of $230.5 \pm 7.3 \mathrm{~g}$ were selected. A total of 12 rats were selected with half male and half female and disinfected on back and abdominal skin, anesthesia with $10 \%$ of hydrochloric acid solution with chloral hydrate by intraperitoneal injection; after succeed in anesthesia, injected $0.5 \mathrm{ml}$ of $10^{7}$ cells / mL UMR-106 osteosarcoma cell line into subcutaneous back locally; applied erythromycin ointment and fed for 2 weeks. Total 4 rats were inoculated successfully. Tissues with no necrosis sarcoma were obtained and cut into $2 \mathrm{~mm} * 2 \mathrm{~mm} * 2 \mathrm{~mm}$ tissue pieces under sterile conditions and transferred into normal saline solution to make tissue suspension. Thirty Sprague-Dawley rats were anesthetized in the same way as above step. $0.2 \mathrm{ml}$ tissue suspension was injected into the back of each rat. Applied with erythromycin ointment at the pinhole and fed for 2 weeks.

Ultrasound Microbubble contrast agent with docetaxel was provided by Jiangsu Hengrui Pharmaceutical Co., Ltd. 
Methods of drug introduction Rats with osteosarcoma were randomly divided into 3 groups with 10 rats in each group. The first group was injected with $1 \mathrm{ml}$ contrast agent with docetaxel (containing docetaxel $0.035 \mathrm{mg}$ ) via caudal vein. Used two-dimensional ultrasound to monitor the image of rat back and irradiated the back of the rat until the contrast agent disappears (About 8 minutes). The applied instrument here were PHILIPS HD11XE, probe L12-3. The second harmonic mode was turned on to make the emission frequency and acceptance frequency at $1.8 \mathrm{MHz}$ and $3.6 \mathrm{MHz}$, respectively. The mechanical index was adjusted to the maximum, and the focus depth was $1 \mathrm{~cm}$. The second group was injected with ultrasound microbubble contrast agent with $0.035 \mathrm{mg}$ docetaxel via tail vein. The third group neither injected drugs nor ultrasound irradiated, used for control. Drug was offered once a week and then widrawed after 5 weeks. The whole body of the rats was observed, and the experimental animals were killed by neck amputation. The animals were fixed on the super clean platform and on the wax plate; opened the skin, took out the whole tumor tissue; observed the tumor volume, weighed the tumor tissue, calculated the tumor inhibition rate, measured the maximum diameter by Vernier caliper. The experimental data was recorded in detail.

Tumor inhibition rate calculation: Tumor inhibition rate $=[($ The average tumor weight of the control group - the mean tumor weight of the drug group) / the average tumor weight of the control group] $\times 100 \%$ [3].

\subsection{Statistical Analysis.}

All data was processed by SPSS17.0 statistical software and $\left(\mathrm{X}^{2} \pm \mathrm{S}\right)$ was used for measured data. The analysis of variance was used among the groups; the LSD method was used when the variance was equal, otherwise, the Dunnetts method was used. There was statistically significant when $\mathrm{P}<0$. 05 .

\section{Results}

The tumor weight of rat with osteosarcoma and the inhibition rate of the experimental results showed that the average weight of osteosarcoma tissue of rats reduced in different degrees by different routes docetaxel administration after treatment. And the differences were statistically significant $(\mathrm{P}<$ 0.05) compared with the control group, among which, the inhibition rates of each experimental group were $24.2 \%, 12.1 \%$ respectively compared with the control group in different ways, see as table 1 .

Table 1. the inhibition rate of animals in each experimental group

\begin{tabular}{cccc}
\hline Experimental group & Group A & Group B & Group C \\
\hline Mean tumor weight & $2.5 \pm 0.12 \mathrm{a}$ & $2.9 \pm 0.11 \mathrm{~b}$ & $3.3 \pm 0.14$ \\
Tumor inhibition rate $(\%)$ & 24.2 & 12.1 & - \\
\hline
\end{tabular}

As compared with group $\mathrm{C}, \mathrm{P}<0.01$; b compared with group $\mathrm{C}, \mathrm{P}<0.05$

\section{Discussion}

Osteosarcoma is a common malignant tumor which mostly occurs in adolescents, and the incidence of osteosarcoma is higest among primary bone tumors. At present, the treatment of osteosarcoma mainly includes surgical treatment, radiotherapy, and chemotherapy and so on. In the past, amputation was the main method of surgical treatment, but with amount of trauma and complications. The surgical method of osteosarcoma supported by neoadjuvant chemotherapy has been improved continuously, gradually becoming the main treatment method of osteosarcoma [4]. Radiotherapy is one of the main treatment methods for the malignant tumor; however, osteosarcoma is not sensitive to radiotherapy and is merely regarded as a general method of postoperative adjuvant treatment. Therefore, adjuvant chemotherapy is a treatment method with greatest potential and prospective. Methotrexate, cisplatin and other drugs are chemotherapeutic drugs with good therapeutic effective, but they are not ideal in the treatment of osteosarcoma. In addition, its role in 
improving the long-term survival is not certain. However, the application of docetaxel, a new chemotherapeutic drug, in the treatment of osteosarcoma has attracted much attention in recent years [5].

Docetaxel is a semisynthetic taxol derivative whose antitumor mechanism is the same as taxol. It promotes polymerization of cell microtubule, and prevents normal physiological microtubule depolymerization, affecting cell mitosis, inhibiting tumor growth and inducing tumor cells apoptosis [6]. It has certain curative effect on breast cancer, ovarian cancer, non-small cell lung cancer, gastric cancer and so on. It is considered to be a broad-spectrum anticancer drug and one of the most effective anticancer drugs at present [7]. However, as a tumor chemotherapeutic drug, docetaxel has low specificity and targeting to malignant tumor cells and normal cells. The normal drug administrated by intravenous injection, the injected docetaxel will distribute along the whole bloody blood circulation system. However only a small amount of docetaxel can reach to the specific tumor tissue at the end; and systemic drug administration may also lead to liver injury, bone marrow suppression, allergic reactions, neurotoxicity and other toxic side effects. Therefore, it has become the focus of research on how to transport docetaxel to osteosarcoma tissue, increase local drug concentration, and reduce drug dosage, thereby reducing adverse reactions, to improve the efficacy, safety and compliance of docetaxel.

The target treatment of drugs carried by ultrasound microbubbles contrast agent is a hot research topic in the current field of medicine, and it can rapidly gather into the target tissue after entering blood circulation. This research successfully adopted the target releasing technology of ultrasound microbubble imaging, and the key to the development of the technology is to improve the transport efficiency of drug carriers, so that they could more enter into the cell to give full play to the efficacy. The main mechanism is that ultrasound irradiation can cause the rupture of contrast agent, which can produce transient transmembrane current on the cell membrane, and further leads to the transient opening of the cell membrane. The extracellular macromolecules seize the opportunity to enter the cell and produce the "acoustic whole effect" [8]. Ultrasound microbubbles carrying with the therapeutic drugs can rupture locally and release therapeutic drugs after ultrasound irradiation, which can obviously enhance its targeting. In this research, docetaxel was connected with ultrasound microbubble surface and injected into rat blood circulation; and ultrasound irradiation was used to destroy microbubble in order to address drug releasing. The satisfactory biological effect was obtained by this method.

To sum up, the research study proved the role of docetaxel in osteosarcoma. The expression of drugs in osteosarcoma tissue was successfully achieved through ultrasound microbubble targeted release technology, resulting in significant decrease tumor growth. Ultrasound microbubble imaging with docetaxel has better targeting and anti-tumor effects than single drug therapy, which provides a possible new therapeutic target for the treatment of osteosarcoma. And because this research study is limited to rat osteosarcoma cells, it is not clear whether the findings of the experiment can be applied to human osteosarcoma, and further research is needed in this area. I also look forward to further follow-up trials that will improve the research vision and open a new door for the treatment of osteosarcoma.

\section{References}

[1]. Yu Zhen, Fan Qing Yu, Hao Xin Bao.Establishment of rat model of pulmonary metastasis of osteosarcoma [J] .Clinical Rehabilitation in China2004,8(20):4048-4049.

[2]. Cu M, Wang Wei, Yu S. Multivariate regression analysis of the survival rate of osteosarcoma [J] .Chinese Journal of Orthopaedic surgery2011, 19(1)36-29.

[3]. Sun Xizang Zhejiang Eat al, Antisense hypoxia-inducible factor-1alpha augments transcatheter arterial embolization in the treatment of hepatocellular carcinomas in rats $[\mathrm{J}]$.Hum Gene Ther.2009 Apr; 20(4):314-24. Doe: 10.1089. 
[4]. Yu Zhuhai, Chen Yu.Clinical analysis of 55 cases of osteosarcoma of extremities [J] .Journal of practical Cancer2008, 23(3): 303-304. [8]Nia Xiaohui, Huang Zhen. Standard chemotherapy of osteosarcoma.

[5]. Huang Xiaocheng, Goo Weighing, Goo Zhen, progress in the treatment of osteosarcoma, medical review2013,6,19(11):1979Medical Review, June 2013, Vol. 19, No. 11.

[6]. Yu Jinxing, Liu Xing, Xu Bo. Docetaxel induced apoptosis of HL-60 / ADR cells and their effects on P-gp and BCL- 2.Effect of BAX protein expression [J] .Chinese Journal of Experimental Hematology2010, 18(2): 311-316.

[7]. Xian Hong. Synthesis and clinical application progress of docetaxel. Tianjin Pharmacology.2008,20(6):68

[8]. Li Kaifeng, Yu Guangdong, Wang Deping. Effects of Nano-hydroxyapatite on the growth of Osteosarcoma U2-OS cells [J] .Journal of Tingyi University2005, 26(1):35-37. 